\title{
A multi-state model for wind farms considering operational outage probability
}

\author{
Lin CHENG ( $\square$ ), Manjun LIU, Yuanzhang SUN, \\ Yi DING
}

\begin{abstract}
As one of the most important renewable energy resources, wind power has drawn much attention in recent years. The stochastic characteristics of wind speed lead to generation output uncertainties of wind energy conversion system (WECS) and affect power system reliability, especially at high wind power penetration levels. Therefore, a more comprehensive analysis toward WECS as well as an appropriate reliability assessment model are essential for maintaining the reliable operation of power systems. In this paper, the impact of wind turbine outage probability on system reliability is firstly developed by considering the following factors: running time, operating environment, operating conditions, and wind speed fluctuations. A multistate model for wind farms is also established. Numerical results illustrate that the proposed model can be well applied to power system reliability assessment as well as solving a series of reliability-centered decision-making problems of power system scheduling and maintenance arrangements.
\end{abstract}

Keywords Wind power, Operational reliability, Outage probability, Multi-state model

Received: 3 October 2012/ Accepted: 26 April 2013/Published online: 12 September 2013

(C) The Author(s) 2013. This article is published with open access at Springerlink.com

L. CHENG, M. LIU, State Key Laboratory of Power Systems,

Department of Electrical Engineering, Tsinghua University,

Beijing 100084, China

$(\bowtie)$ e-mail: chenglin@mail.tsinghua.edu.cn

M. LIU

e-mail:1mj06@mails.tsinghua.edu.cn

Y. SUN, School of Electrical Engineering, Wuhan University,

Wuhan, China

e-mail: yzsun@tsinghua.edu.cn

Y. DING, Department of Electrical Engineering, Technical

University of Denmark, Kongens Lyngby, Denmark

e-mail: yding@elektro.dtu.dk

\section{Introduction}

Along with the rapid development of wind power technologies, the wind power penetration level grows continuously in power systems around the world. However, it also leads to the higher uncertainties and risks of power system operation and planning.

Recently, power system reliability evaluation is worldwidely recognized due to risks caused by wind power integration. Therefore, accurately and authentically modeling the uncertainty of wind energy conversion system (WECS) for improving real-time power system reliability performance has become a new research focus.

A reliability evaluation model incorporating with wind power is proposed $[1,2]$, considering the wind speed prediction model and generation output characteristic model of wind turbines. Among these studies, the wind turbine outage rate is usually regarded as a constant value.

In traditional power system reliability model, the studied time period is usually a year or longer for the planning purposes rather than power system operation, thus serving for the power system operation. The basic concepts of operational reliability are proposed initially in [3], and its basic point is that the reliability level of a system varies with changes under operating conditions of a power system. The operational reliability is based on the time-varying reliability model of system components, which requires their parameters to reflect changes of the system operating conditions.

The reliability model of components is established in [3] based on the real-time operating conditions considering the impact of the components outage probability of the line flow, bus voltage and system frequency, etc. With the model, it is able to assess the reliability level of a fault state or an abnormal operation state. References $[4,5]$ studied the impact of ambient temperature and load rate on aging 
failure rate of transformers. References [6-11] analyzed the factors that cause component outage and established an element outage rate model based on multiple operating conditions. The above researches mainly focused on establishing component function of outage probability or outage rate that using operating conditions as the independent variables. However, they did not take into account the impact of time scale on the component outage, making it difficult to reflect the reliability level of the short-term power system operation. Therefore, the operational reliability assessment theory, which has the ability to reflect the effects due to a series of elements (health status of elements, operating environment and operating conditions), can be adopted to guide decision makings (planning, operation and maintenance) with much more comprehensive information. However, the operational reliability assessment of wind power is still in the start-up phase. References [12,13] analyzed the correlation between the outage rate and the wind speed, and established a wind power variation model considering of outage rate which is dependent upon wind speed.

References [14, 15] proposed a multi-state model, which has been proved to be more reasonable than traditional two-state generator models. However, time varying factors, such as outages, cannot be reflected in conventional multistate models. In this paper, we focus on modifying the existing multi-state WECS model to reflect time varying factors by adopting operational reliability theories.

\section{Operational outage probability model of wind turbines}

The power system operational reliability is defined by considering components' own health, external environmental conditions, system operating conditions, and system operational behavior, the ability of power systems to continuously provide electricity and energy to customers with an acceptable quality standards and the number of need in a short time [7]. The short-term outage probability model of wind turbines can be divided into four parts based on the previous description.

\subsection{Time-dependent aging outage model}

A large number of outage data of electrical components show that the outage rate of the component follows the life basin curve, and has three distinct areas [16], as shown in Fig. 1.

Area I represents the commissioning period, in which the component outage rate has decreased over time; Area II represents the effective life period in which the component outage rate is almost constant; Area III is for the

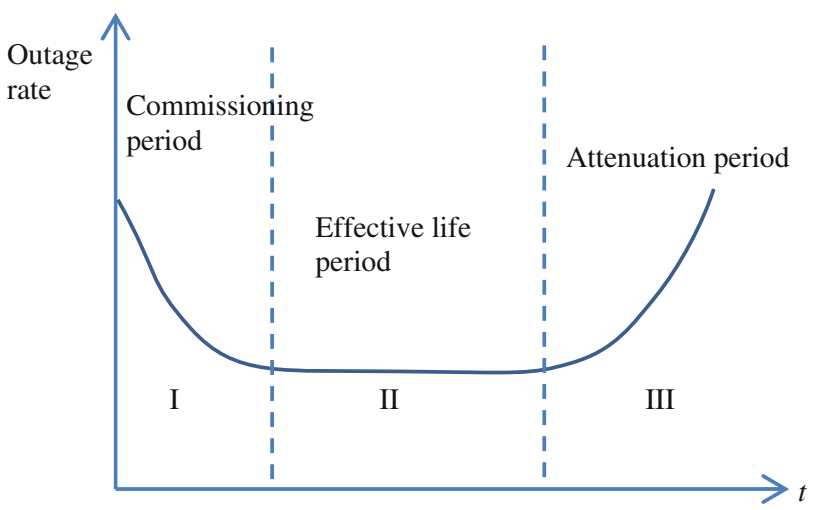

Fig. 1 Life basin curve

attenuation period, in which the component outage rate has an increasing trend as the time passing by. Component aging outage that occurred in the attenuation period is related to the history (that is, the component service time) and conditional outage events.

The aging process usually follows the Weibull distribution, and the functions of outage rate and cumulative probability can be represented as

$\lambda_{\mathrm{a}}(t)=\frac{\beta}{\eta}\left(\frac{t}{\eta}\right)^{\beta-1}$,

$F_{\mathrm{a}}(t)=1-\mathrm{e}^{-\left(\frac{t}{\eta}\right)^{\beta}}$,

where $t$ is the operating time, $\beta$ is a shape parameter and $\eta$ is the scale parameter, also known as the characteristic life parameters.

According to the definition of conditional probability, after the component has worked for $T$ hours, its outage probability during the interval $\Delta t[8]$ is

$P_{\mathrm{a}}=\operatorname{Pr}(T \leq t \leq T+\Delta t \mid t>T)=\frac{F_{\mathrm{a}}(T+\Delta t)-F_{\mathrm{a}}(T)}{1-F_{\mathrm{a}}(T)}$.

Substituting (2) into (3), it can be obtained that

$P_{\mathrm{a}}=1-\mathrm{e}^{\left(\frac{T}{\eta}\right)^{\beta}-\left(\frac{T+\Delta t}{\eta}\right)^{\beta}}$.

\subsection{Weather-dependent random outage model}

In addition to aging outage model, there are many unpredictable factors affecting the forced outage rates of wind turbines, such as the inappropriate operation, improper maintenance, lightning and snow disaster. As the wind turbines are exposed to the air, the outage rate is closely related to the weather conditions. In the cases of extremely bad weather conditions, such as thunderstorms, typhoons, hurricanes, snow and ice, the outage rate will greatly increase. Reference [17] used two state model (normal 
weather and bad weather) or multi-state model to describe the weathers. For simplicity, this paper uses two-state weather model to describe the occasional outage rate of wind turbines, it can be written as

$\lambda_{\mathrm{c}}(w)=\left\{\begin{array}{ll}\bar{\lambda} \frac{N+S}{N}(1-F), & w=0 \\ \bar{\lambda} \frac{N+S}{S} F, & w=1\end{array}\right.$,

where $\bar{\lambda}$ is the average outage rate of wind turbines occasional outage rate, $N$ is the duration of normal weather, $S$ is the duration of bad weather, $F$ is the proportion of outage rate in bad weather, $w$ is the current weather conditions, and $w=0$ stands for normal weather while $w=1$ stands for bad weather.

If the weather condition does not change within a short time $\Delta t$, then the outage rate is unchanged. The occasional outage probability that wind turbines fail at $\Delta t$ follows the exponential distribution:

$P_{\mathrm{gc}}=1-\mathrm{e}^{-\lambda_{\mathrm{gc}} \Delta t}$

where $\lambda_{\mathrm{gc}}$ is the occasional outage probability.

\subsection{Current-dependent overload protection outage model}

One component's overload tripping will lead to other components' tripping, which will result in a cascading failure. Similar to conventional generators, wind turbines have overload protection, however, the trigging value error of protection device will lead to protection uncertainty.

Let the error of protection system trigger current value, $I_{\text {set }}$ be $\pm \varepsilon$, then follow the truncated normal distribution with an average of $I_{\text {set0 }}$, and a standard deviation of $\sigma$, range from $I_{\text {set } 0}(1-\varepsilon)$ to $I_{\text {set } 0}(1+\varepsilon)$. A density function is as follows:

$$
\begin{aligned}
& f\left(I_{\mathrm{set}}\right) \\
& \quad=\left\{\begin{array}{l}
0, I_{\mathrm{set}} \notin\left[I_{\mathrm{set} 0}(1-\varepsilon), I_{\mathrm{set} 0}(1+\varepsilon)\right] \\
\frac{1}{a \sigma \sqrt{2 \pi}} \exp \left[-\frac{\left(I_{\mathrm{set}}-I_{\mathrm{set} 0}\right)^{2}}{2 \sigma^{2}}\right], I_{\mathrm{set}} \in\left[I_{\mathrm{set} 0}(1-\varepsilon), I_{\mathrm{set} 0}(1+\varepsilon)\right],
\end{array}\right.
\end{aligned}
$$

$a=\phi\left(\frac{\varepsilon I_{\mathrm{set} 0}}{\sigma}\right)-\phi\left(\frac{-\varepsilon I_{\mathrm{set} 0}}{\sigma}\right)$

where $\phi$ is the standard normal distribution function.

Let $I$ be the component current, event $A=\{$ The component resection made by protection action $\}$, event $B_{1}=\left\{I \geq I_{\text {set }}\right\}$, event $B_{2}=\left\{I<I_{\text {set }}\right\}$, then:

$$
P(A)=P\left(A \mid B_{1}\right) P\left(B_{1}\right)+P\left(A \mid B_{2}\right) P\left(B_{2}\right)
$$

where $P(A)$ is the outage probability made by overload protection action, written as $P_{\mathrm{r}}, P\left(A \mid B_{1}\right)$ is the probability of protection right action, written as $P_{\mathrm{z}}$, and $P\left(A \mid B_{2}\right)$ is the probability of protection malfunction, written as $P_{\mathrm{w}} . P_{\mathrm{z}}$ and $P_{\mathrm{w}}$

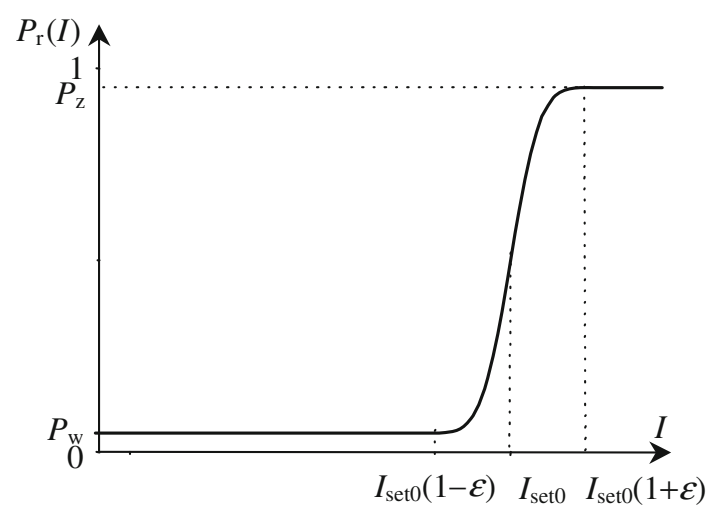

Fig. 2 Model of overload protection action

can be obtained through statistical analysis.Based on (9), when $I<I_{\text {seto }}(1-\varepsilon), P\left(B_{1}\right)=0, P\left(B_{2}\right)=1$, it can be obtained that $P_{\mathrm{r}}(I)=P_{\mathrm{w}}$.

When $I>I_{\text {set } 0}(1+\varepsilon), P\left(B_{1}\right)=1, P\left(B_{2}\right)=0$, it can be obtained that

$P_{\mathrm{r}}(I)=P_{\mathrm{z}}$.

When $I_{\text {set } 0}(1-\varepsilon) \leq I \leq I_{\text {set } 0}(1+\varepsilon)$, it can be obtained that

$P_{\mathrm{r}}(I)=P_{\mathrm{z}} \int_{I_{\mathrm{set} 0}(1-\varepsilon)}^{I} f\left(I_{\mathrm{set}}\right) \mathrm{d} I_{\mathrm{set}}+P_{\mathrm{w}} \int_{I}^{I_{\text {set } 0}(1+\varepsilon)} f\left(I_{\text {set }}\right) \mathrm{d} I_{\text {set }}$

Equations (10)-(12) are described as shown in Fig. 2.

\subsection{Wind speed-dependent outage model}

Through the statistical analysis on the measured data of wind power, [12] obtained the outage probability statistics of wind turbines, which follows the changes of wind speed,

Table 1 Statistics of outage probabilities of wind turbines with normal wind speed

\begin{tabular}{ll}
\hline Wind speed $(\mathrm{m} / \mathrm{s})$ & Outage probability \\
\hline 8 & 0.012707 \\
9 & 0.017897 \\
10 & 0.025492 \\
11 & 0.027043 \\
12 & 0.036700 \\
13 & 0.044444 \\
14 & 0.053561 \\
15 & 0.077190 \\
16 & 0.104439 \\
17 & 0.133333 \\
18 & 0.163728 \\
19 & 0.165436 \\
20 & 0.240741 \\
\hline
\end{tabular}




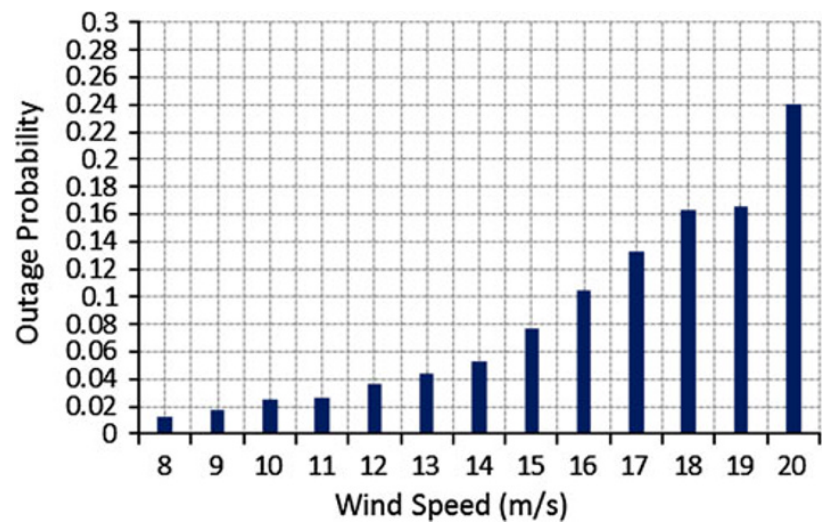

Fig. 3 Wind speed-dependent outage probability of wind turbines

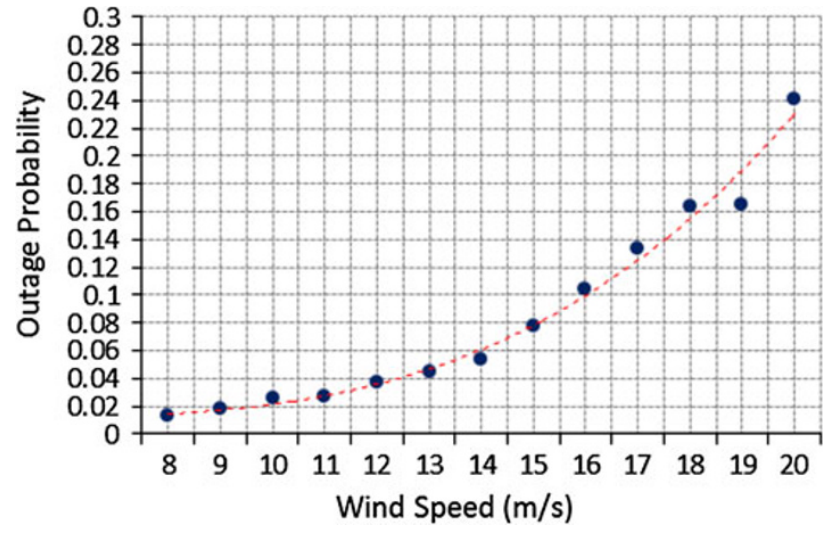

Fig. 4 Curve fitting of the outage probability versus wind speed

as shown in Table 1 . The outage probability of wind turbines versus wind speed is also shown in Fig. 3.

For a better description of the relationship between outage probability and wind speed, a cubic function is used to fit the curve as shown in Fig. 4.

The cubic fitting function can be represented as

$P_{\mathrm{gv}}(v)=a v^{3}+b v^{2}+c v+d$,

where $P_{\mathrm{gv}}(v)$ is the outage probability, $v$ is the wind speed, $a, b, c$, and $d$ are coefficins.

As a result, it is possible to obtain a simple wind speeddependent outage probability model, which is consistent with the actual situation. With a prolonged outside exposure, the wind turbine generation output is directly related to the wind speed. It indicates that the occasional outage of wind turbines is correlated to the weather conditions, while the ordinary outage probability is related to wind speed.

\subsection{Hybrid outage probability model}

For wind turbines, aging outage, occasional outage and outage caused by overload protection action and wind speed-dependent outage are independent under certain conditions. Therefore, the short-term reliability model of wind turbines can be expressed as

$P_{\mathrm{g}}=1-\left(1-P_{\mathrm{ga}}\right)\left(1-P_{\mathrm{gc}}\right)\left(1-P_{\mathrm{gv}}\right)\left(1-P_{\mathrm{r}}\right)$,

where $P_{\mathrm{g}}$ is the wind turbines outage probability; $P_{\mathrm{ga}}$ the aging outage; $P_{\mathrm{gc}}$ the occasional outage; $P_{\mathrm{gv}}$ the wind speed-dependent outage probability; and $P_{\mathrm{r}}$ is the overload protection caused outage.

\section{Multi-state model of wind farm}

Statistical data indicate that many generators cannot always work at its rated state, meanwhile, they seldom enter full outage state as well. In fact, working at one or more de-rated states is also a common scene. In this way, a concept of derating-adjusted forced outage rates (DAFOR) is proposed to describe the derated state [13], and then an apportioning method is used to calculate the derated state and its outage probability [14].

Compared with the modeling of conventional generators, multi-state modeling process of wind farms have many differences. The output of the wind farm is directly related to wind speed, presenting a strong stochastic property, therefore, the multi-state model of the wind farm at different wind speeds cannot be uniformed, thus containing respective probability distribution characteristics. A model for estimating the wind power variation by modeling the outage of wind turbines is proposed in [15]. Considering the operational outage probability model of wind turbines, the multi-state model of wind farms at different wind speed segments is modeled respectively. The wind farm analyzed in this paper is located in Hebei Province, China. All of the wind turbines are supposed to work in an effective life period, and the overload protection outage is ignored for the reason and the power output is lower than its rated capacity based on the historical statistics. To simplify the process, the average wind speeds of 5 and $10 \mathrm{~m} / \mathrm{s}$ are adopted, the wind speed below $7.5 \mathrm{~m} / \mathrm{s}$ is taken as $5 \mathrm{~m} / \mathrm{s}$, while wind speed over $7.5 \mathrm{~m} / \mathrm{s}$ is taken as $10 \mathrm{~m} / \mathrm{s}$. Therefore, the output distribution at different wind speed levels can be obtained as follows.

1) Average wind speed of $10 \mathrm{~m} / \mathrm{s}$

Figure 5 shows that at the average wind speed of $10 \mathrm{~m} /$ $\mathrm{s}$, the output of wind farm considering the outage probability is more in line with the actual situation.

Considering the outage probability, the power output of the wind farm at the average wind speed of $10 \mathrm{~m} / \mathrm{s}$ can be decomposed into five states with the output level of 0.1, 0.35, 0.5, 0.7, 0.9, respectively, as shown in Fig. 6. Unlike conventional generators, considering wind speed 


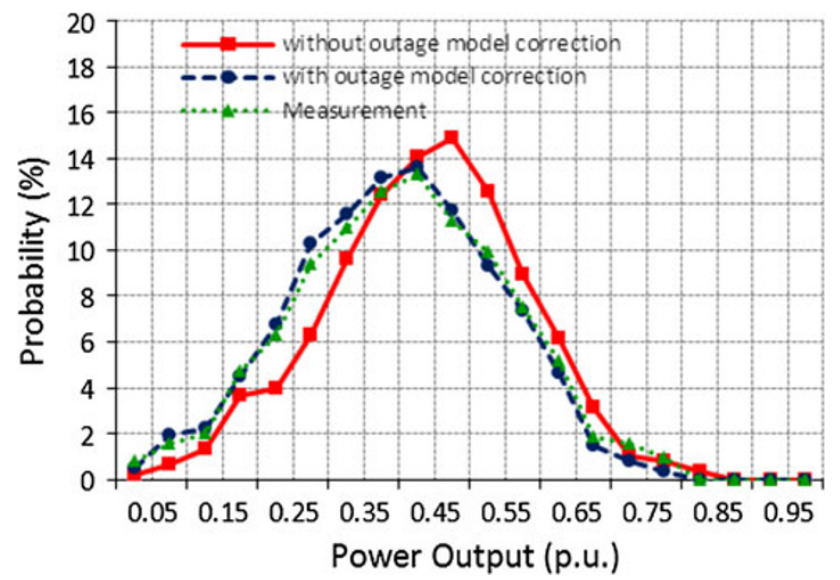

Fig. 5 Power output of wind farm at average wind speed of $10 \mathrm{~m} / \mathrm{s}$

uncertainty and output fluctuation characteristics of wind turbines at different wind speed levels, the equivalent of a multi-state model for the wind farm is not decomposed from rated capacity to outage state, but to choose 5 larger probabilities states as representatives. This phenomenon is more obvious with the 5-state model at the average wind speed of $5 \mathrm{~m} / \mathrm{s}$.

2) Average wind speed of $5 \mathrm{~m} / \mathrm{s}$

Similar to the above analysis, the power output distribution under an average wind speed of $5 \mathrm{~m} / \mathrm{s}$ (as shown in Fig. 7) can also be obtained. As the wind speed is close to cut-in speed of the wind turbines, the generation output of wind turbines is relatively low. As shown in Fig. 8, there're five kinds of power output representatives with the highest occurrence probabilities, the corresponding output of 0.05 , $0.1,0.15,0.2$ and 0.25 of the rated output are selected in order to describe the situation more accurately, since a linear output steps would result in a series of useless output states with extremely small probabilities.

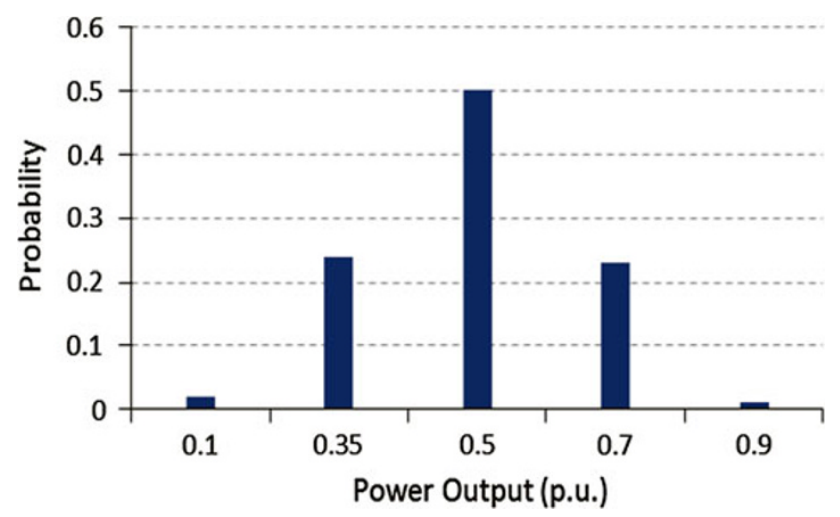

Fig. 6 5-state model for wind farm at average wind speed of $10 \mathrm{~m} / \mathrm{s}$

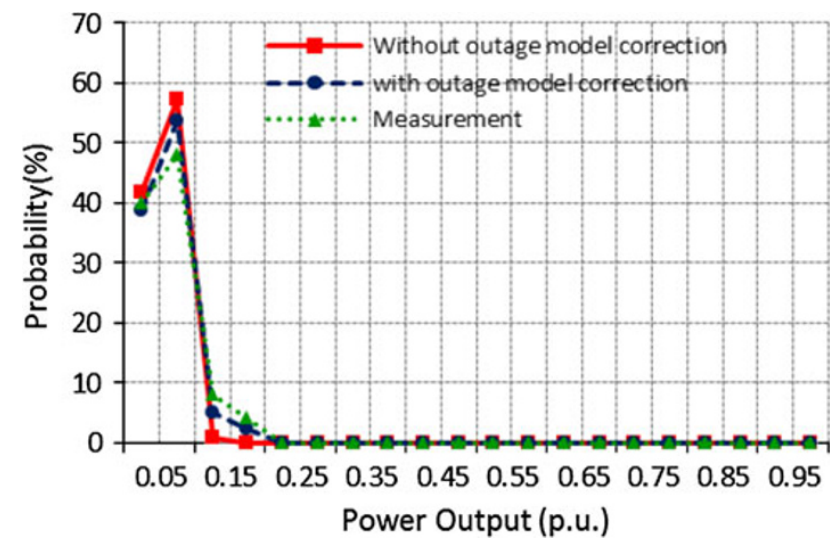

Fig. 7 Power output of wind farm under average wind speed of $5 \mathrm{~m} / \mathrm{s}$

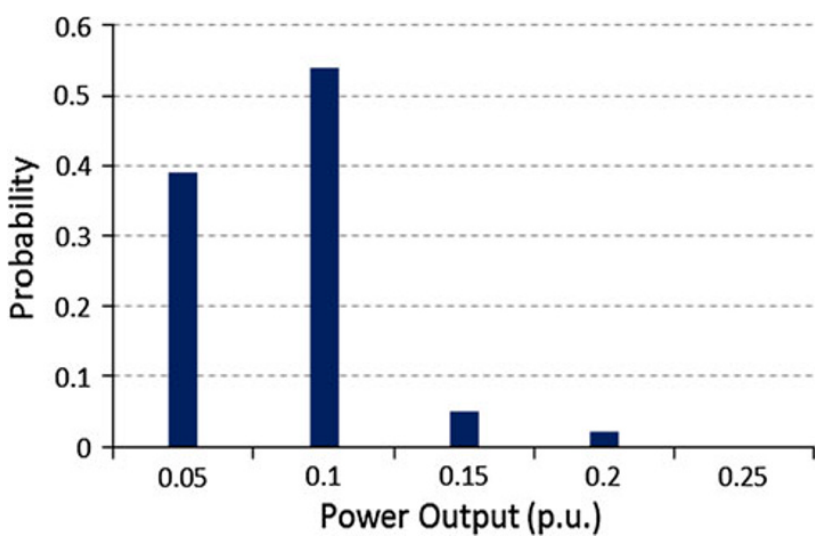

Fig. 8 5-state model of wind farm under average wind speed of $5 \mathrm{~m} / \mathrm{s}$

\section{Case study}

\subsection{System introduction}

This section discuss the significance of the multi-state model, considering the operational outage probability based on the reliability indices calculated from the improved RTS system [18]. The system contains 24 buses, 32 generators, with a total capacity of 3,405 MW, and the wind farm is supposed to be connected to the system in bus 14, as shown in Fig. 9.

Two situations in which the capacity of wind farm is $900 \mathrm{MW}$ (a penetration of $20.9 \%$ ) and 2,200 MW (a penetration of $5.5 \%$ ) are taken into account. For each situation, 4 cases are analyzed.

Case A (optimistic case): The forecasted wind production potential is considered to be $100 \%$ reliable.

Case B (pessimistic case): The forecasted wind production potential is completely unreliable.

Case $\mathrm{C}$ : The forced outage rate (FOR) of wind turbines is considered $(\mathrm{FOR}=0.10)$. 
Case D: The multi-state model considers operational reliability outage probability.

It is assumed that the system unit commitment schedule as well as the load forecasting data and wind speed forecast data are known, and then the wind turbine output can be calculated. The 24-hour online capacity and load are shown in Fig. 10.

Meanwhile, the forecasted 24-hour wind speed and power output can be obtained, as shown in Fig. 11.

\subsection{System operational reliability assessment}

under different wind power penetration

In order to compare the reliability of the system in different wind power penetration, the reliability indices of Cases A-D

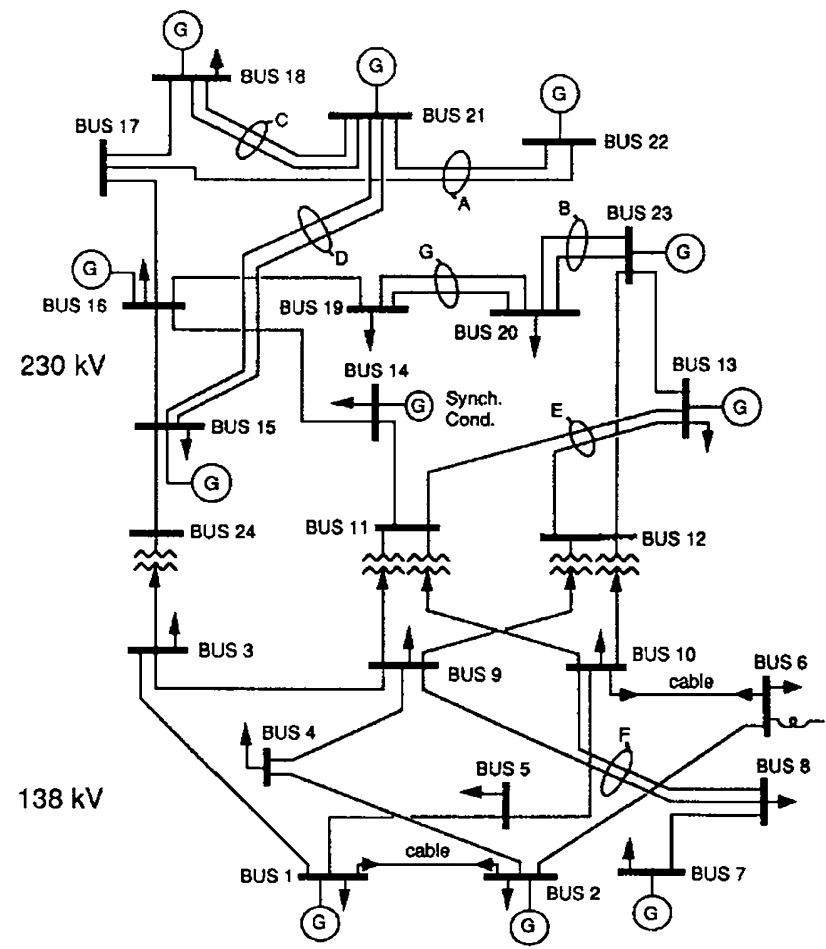

Fig. 9 Diagram of IEEE reliability test system

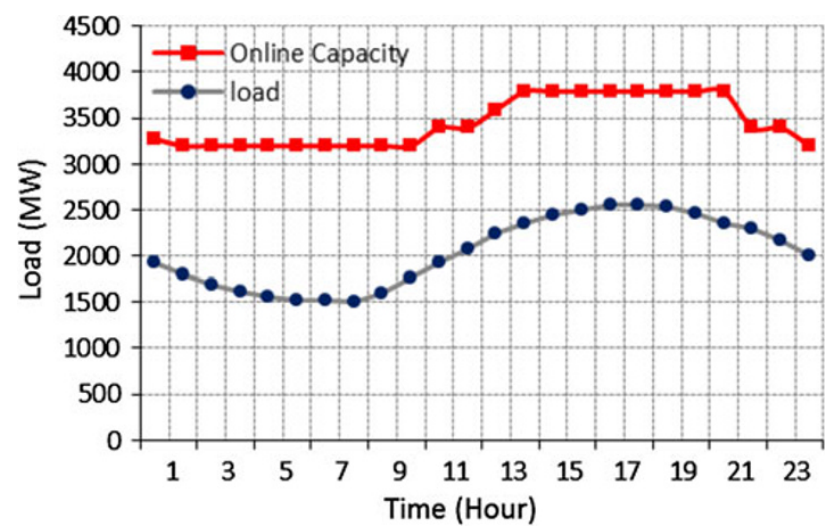

Fig. 10 24-hour unit commitment and load are calculated at two different penetration levels of $20.9 \%$ and $5.5 \%$, the results are shown in Figs. 12, 13, 14, 15.

By contrast analysis, when the wind power penetration is low, the integration of wind power in power system has little impact on the system reliability, where LOLP and LOEE maintained at a high level. With the increase of wind power penetration, the impact of wind power on power

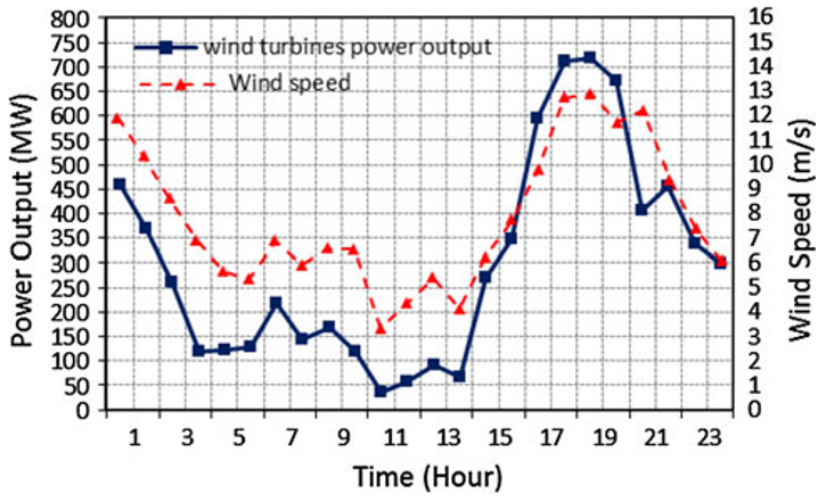

Fig. 11 24-hour wind turbine output

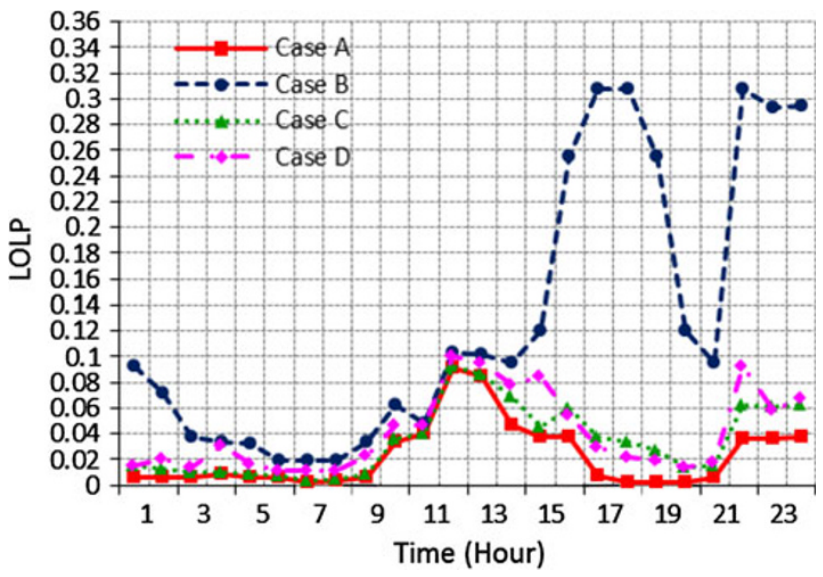

Fig. 12 Loss of load probability (LOLP) under $20.9 \%$ penetration

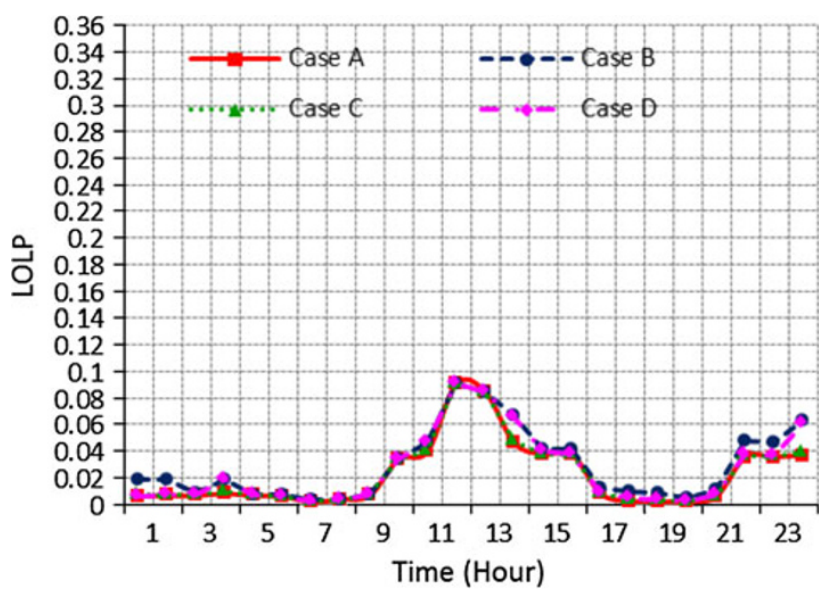

Fig. 13 The LOLP under $5.5 \%$ penetration 


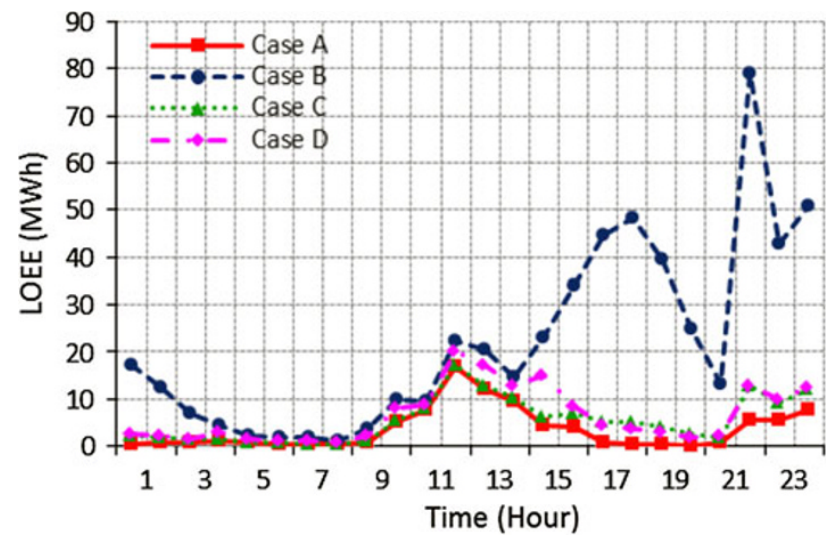

Fig. 14 Loss of energy expectation (LOEE) under $20.9 \%$ penetration

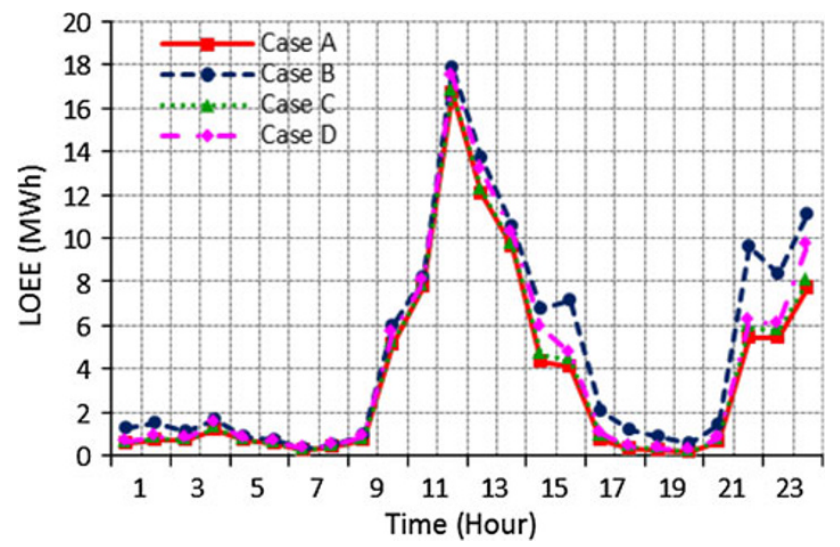

Fig. 15 LOEE under $5.5 \%$ penetration

system reliability increases, as shown in Fig. 12. When wind power penetration is $20.9 \%$, the maximum LOLP of the system will reach up to 0.308 without the integration of wind power, while the LOLPs are 0.02891 considering a constant FOR of wind turbines and 0.02890 considering the operational outage probability, respectively. Reliability level will be declined by more than 10 times. Similarly, the LOEE of system without the integration of wind power is 6 times higher than that considering operational outage probability model, as shown in Fig. 14. Therefore, with the increasing of wind power penetration, it will have a significant impact on the power system reliability.

\subsection{Difference between multi-state and two-state models}

At the same time, the effect of multi-state on the system operational reliability is analyzed at different wind speeds. By observing Fig. 12, where Case $\mathrm{C}$ uses the two-state model with a constant outage probability while Case D adopts the five-state wind farm model. As a result, the five-state model has a more pessimistic result compared with the two-state model, but it is more suitable to reflect the relationship between wind power output and wind speed. As for the reliability index of 4:00 and 15:00 shown in Fig. 12, it can be explained that when the wind speed is low, it is not enough to start the wind turbine. And the wind turbine will stay in outage state, finally, the system operational reliability will decline.

\subsection{Impact of wind speed and load changes on system operational reliability}

Assuming that the wind speed prediction and wind turbine output are known, the system operational reliability is analyzed at a specific state, such as that at 18:00 when the load is increasing while the unit commitment is unchanged. The forecast shows that at 18:00, the wind turbines' output will increase to meet the increased load demand, the system is maintained at a higher level of reliability. Therefore, in this case, operators can arrange conventional unit overhaul and to make a reasonable planning unit commitment for the next hour. However, at 24:00, wind turbine output will decrease, while there is a short decline at the level of system load, but the decreased amount of load is less than the decreased amount of unit output, and it will end with a drop of the operational reliability.

\subsection{Impact of unit commitment change on operational reliability}

The assessment of operational reliability plays an important role in power system unit commitment schedule. As shown in Fig. 11, at 22:00, power output of the wind turbines increase modestly and the load level slightly changes, originally in unit commitment, units that put into operation are reduced, thus leading to the lower level of operational reliability of the system. If increasing unit output at 22:00, the system reliability level will significantly increase, as shown in Fig. 16, which is of great significance for guiding operation scheduling.

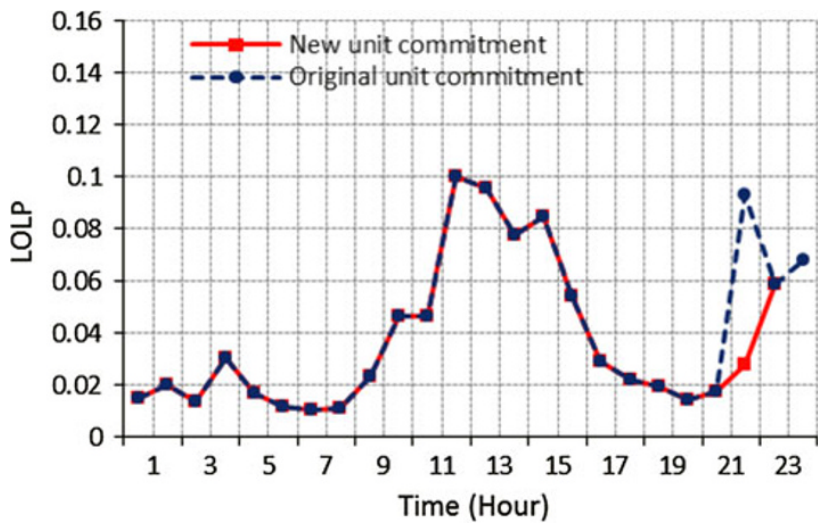

Fig. 16 Impact on system reliability index caused by adding spinning reserve 


\section{Conclusion}

Starting from the two aspects of the operational conditions and the operational time, the proposed model comprehensively analyzes the outage probability of wind turbines in actual operation. Based on a multi-state model of the conventional generators, this paper presents a multi-state model of wind farm considering of operational outage probability. The multi-state model is considered at different average wind speed levels, respectively, which can better reflect the actual operating conditions of the wind turbines.

At last, the paper elaborates the significance of the operation reliability model for operation scheduling by a practical example. With data of wind speed forecast and load forecast, the model can play a guiding role in system operation scheduling, maintenance arrangement and unit commitment scheduling.

However, in this model, different wind speed levels correspond to different multi-state models, the states of the system will exponentially increase with an increasing of system scale, which brings new challenge to the time and efficiency of the assessment. Therefore, how to achieve rapid assessment of the operational reliability for wind power is a next focus of research.

Acknowledgments This research work was supported by National High Technology Research and Development Program of China (“863” Program) (No. 2011AA05A103).

Open Access This article is distributed under the terms of the Creative Commons Attribution License which permits any use, distribution, and reproduction in any medium, provided the original author(s) and the source are credited.

\section{References}

[1] Billinton R, Chen H, Ghajar R (1996) Time-series models for reliability evaluation of power systems including wind energy. Microelectron Reliab 36(9):1253-1261

[2] Karki R, Po H, Billinton R (2006) A simplified wind power generation model for reliability evaluation. IEEE Trans Energy Conver 21(2):533-540

[3] Sun Y, Cheng L, Liu H et al (2005) Power system operational reliability evaluation based on real-time operating state. In: Seventh international power engineering conference, Singapore

[4] He J, Cheng L, Sun Y (2007) Transformer real-time reliability model based on operating conditions. J Zhejiang Univ Sci A 8(3):378-383

[5] He J, Sun Y, Wang P et al (2009) A hybrid conditions-dependent outage model of a transformer in reliability evaluation. IEEE Trans Power Deliver 24(4):2025-2033

[6] Cheng L, Liu H, Zou X et al (2007) Short-term reliability online evaluation basing on transient state probability. In: Power engineering society general meeting, Florida, USA
[7] Sun Y, Cheng L, He J (2012) Power system operational reliability theory. Tsinghua University Press, Beijing

[8] Sun Y, Cheng L, Ye X et al (2010) Overview of power system operational reliability. In: 11th probabilistic methods applied to power systems, Singapore

[9] Wang P, Billinton R (2002) Reliability cost/worth assessment of distribution systems incorporating time-varying weather conditions and restoration resources. IEEE Trans Power Deliv 17(1):260-265

[10] Sun Y, Wang P, Cheng L et al (2010) Operational reliability assessment of power systems considering condition-dependent outage rate. IEE Proc Gener Transm Distrib 4(1):60-72

[11] Billinton R, Li W (1991) A novel method for incorporating weather effects in composite system adequacy evaluation. IEEE Trans Power Syst 6(3):1154-1160

[12] Liu M, Cheng L, Ye X et al (2012) Wind turbine generator reliability model considering wind-speed dependent outage rate. In: 12th probabilistic methods applied to power Systems, Turkey, Japan

[13] Billinton R, Gao Y (2008) Multistate wind energy conversion system models for adequacy assessment of generating systems incorporating wind energy. IEEE Trans Energy Convers 23(1): $163-170$

[14] Billinton R, Li Y (2004) Incorporating multi-state unit models in composite system adequacy assessment. In: 8th international conference on probabilistic methods applied to power systems, Ames, Iowa, USA

[15] Cheng L, Lin J, Sun Y et al (2012) A model for assessing the power variation of a wind farm considering the outages of wind turbines. IEEE Trans Sustain Energy 3(3):432-444

[16] Li W (2005) Risk assessment of power systems: models, methods, and applications. IEEE Press/Wiley, New York

[17] Billinton R, Allan RN (1996) Reliability evaluation of power systems, 2nd edn. Plenum Press, New York

[18] Grigg C, Wong P, Albrecht P et al (1999) The IEEE reliability test system-1996: a report prepared by the reliability test system task force of the application of probability methods subcommittee. IEEE Trans Power Syst 14(3):1010-1020

\section{Author Biographies}

Lin CHENG (M'05) was born in 1973. He received the B.S. degree from Tianjin University, Tianjin, China, in 1996 and the Ph.D. degree in electrical engineering from Tsinghua University, Beijing, China, in 2001. Currently, he is an Associate Professor with the State Key Laboratory of Power Systems, Department of Electrical Engineering, Tsinghua University. His research interests are in the areas of power system reliability and planning, power system dynamics and control, as well as voltage stability and control.

Manjun LIU was born in 1987. He received his Bachelor degree in Department of Electrical Engineering from Tsinghua University, China in 2010. He is now pursuing a Ph.D. degree in the same department. His research interest is the reliability of power systems incorporating with intermittent electrical source.

Yuanzhang SUN (M'99, SM'01) received the B.S. degree from Wuhan University of Hydro and Electrical Engineering, China, in 1978, the M.S. degree from Electric Power Research Institute (EPRI), China, in 1982, and $\mathrm{Ph}$. D. degree from Tsinghua University, China, in 1988, all in electrical engineering. He is now a chair professor at Tsinghua University and Wuhan University, China. His main research interests include power systems dynamics and control, voltage stability and control, and renewable energy. 
Yi DING is an Associate Professor in the Department of Electrical Engineering, Technical University of Denmark (DTU), Denmark. He received the B. Eng. degree from Shanghai Jiao Tong University, China, and the Ph.D. degree from Nanyang Technological University,
Singapore, both in electrical engineering. His research interests include power systems reliability/performance analysis incorporating renewable energy resources, smart grid performance analysis, and engineering systems reliability modeling and optimization. 